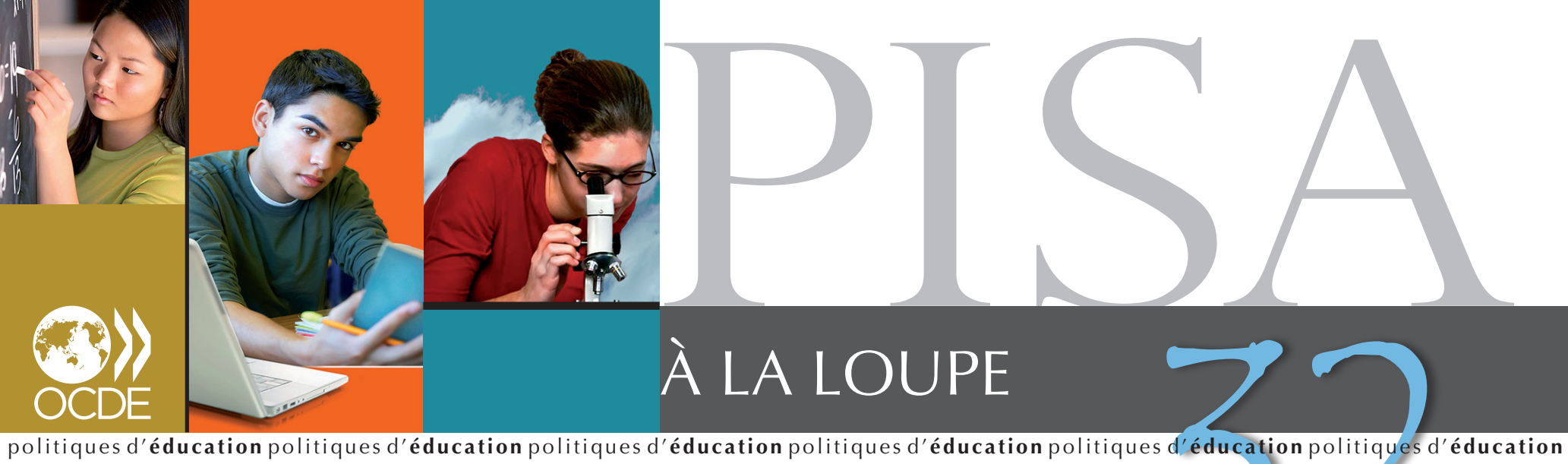

politiques d'éducation politiques d'éducation politiques d'éducation politiques d'éducation politiques d'éducation politiques d'éducation

\title{
Les élèves sont-ils plus performants dans les établissements où règne un bon climat de discipline?
}

- La plupart des élèves bénéficient d'un bon climat de discipline en classe durant leurs cours de la langue d'enseignement.

- Les élèves issus d’un milieu socio-économique défavorisé sont moins susceptibles que les élèves issus d’un milieu socio-économique favorisé de bénéficier d’un bon climat de discipline en classe.

- Un bon climat de discipline en classe - indépendamment du profil socio-économique global de l'établissement - est associé à un meilleur niveau de performance.

Presque partout, les classes offrent un climat propice à l'apprentissage.
Imaginez une classe d'élèves de 15 ans. Quelle image vous vient spontanément à

l'esprit? Des rangées bien ordonnées de bureaux où sont assis des élèves buvant, dans la plus grande passivité et un silence religieux, chaque parole de leur enseignant? Ou un semi-chaos où les élèves bavardent - ou s'interpellent - à travers la classe face à un enseignant impuissant tentant désespérément d'attirer leur attention? Fort heureusement, la réalité se situe quelque part entre ces deux extrêmes. Selon les déclarations des élèves qui ont participé à l'enquête PISA 2009, la majorité des élèves de la plupart des pays et économies participant au PISA bénéficient d'un bon climat de discipline en classe. Ainsi, en moyenne, dans les pays de l'OCDE, quatre élèves sur cinq déclarent qu'il n'arrive que rarement qu'ils ne puissent pas bien travailler en classe à cause des autres élèves ; environ trois élèves sur quatre déclarent par ailleurs qu'il n'arrive que rarement que leurs enseignants aient à attendre un long moment avant que leurs élèves se calment; et plus de deux élèves sur trois déclarent qu'il n'y a jamais ou presque jamais de bruit et d'agitation en classe.

Certains pays et économies offrent un climat de discipline encore plus propice à I'apprentissage. Ainsi, en Corée et en Thaïlande, moins d'un élève sur dix déclare qu'il ne peut pas bien travailler en classe à cause du désordre ; au Japon, au Kazakhstan et en Thaïlande, moins d'un élève sur dix déclare qu'il ne commence à travailler que bien après le début du cours ; et enfin, au Japon, au Kazakhstan et à Shanghai (Chine), moins d'un élève sur dix déclare que son enseignant doit attendre un long moment avant que les élèves se calment pour pouvoir commencer son cours. 


\section{La discipline en classe}

Pourcentage d'élèves ayant déclaré que les situations suivantes ne surviennent « jamais ou presque jamais ou qu' « à quelques cours » lors de leurs cours de la langue d'enseignement

$\checkmark$ Les élèves ne peuvent pas bien travailler.

- L'enseignant doit attendre un long moment avant que les élèves se calment.

- Il y a du bruit et de l'agitation.

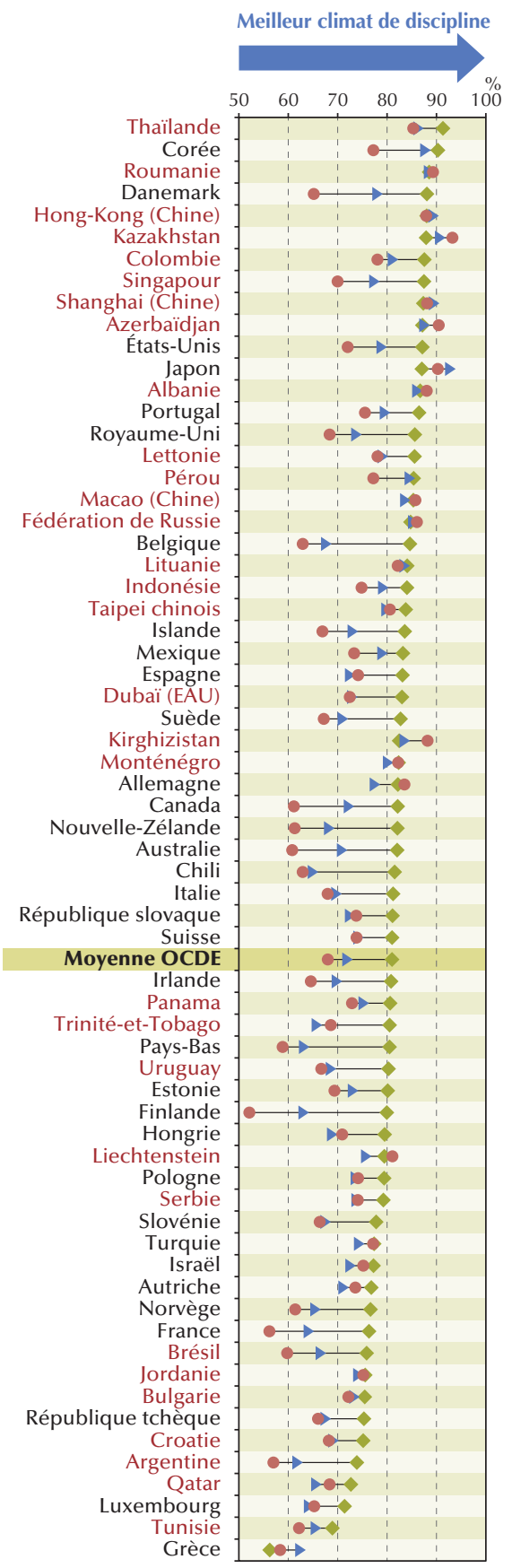

Remarque : les pays de l'OCDE sont indiqués en noir.

Source : Résultats du PISA 2009: Les clés de la réussite des établissement d'enseignement : Ressources, politiques et pratiques, figure IV.4.2.

StatLink 年II $\mathrm{http}: / / \mathrm{dx}$.doi.org/10.1787/888932343418
Par contraste, en Grèce, près d'un élève sur deux déclare ne pas pouvoir bien travailler dans la plupart, voire la totalité, des cours : en Croatie, en Grèce et en Slovénie, près d'un élève sur deux déclare que les élèves n'écoutent pas ce que dit l'enseignant ; et enfin, en Argentine, en Finlande, en France, en Grèce et aux Pays-Bas, près d'un élève sur deux déclare qu'il y a du bruit et de l'agitation en classe.
Lors du cycle PISA 2009, les élèves ont été invités à répondre aux questions suivantes :

\section{Durant vos cours de la langue d'enseignement, à quelle fréquence les situations suivantes se produisent-elles ?} (Ne cochez qu'une seule case par ligne)

$\begin{array}{cccc}\text { Jamais } & \grave{A} & \grave{A} / a & \\ \text { ou } & \text { quelques } & \text { plupart } & \text { Àtous } \\ \text { presque } & \begin{array}{c}\text { cours } \\ \text { jamais }\end{array} & \text { descours } & \end{array}$

a) Les élèves n'écoutent pas ce que dit l'enseignant.

b) Il y a du bruit et de l'agitation.

c) L'enseignant doit attendre un long moment avant que les élèves se calment.

d) Les élèves ne peuvent pas bien travailler.

e) Les élèves ne commencent à travailler que bien après le début du cours.

Les déclarations des élèves sur la mesure dans laquelle le climat de leur classe leur semble propice à l'apprentissage ont été combinées afin d'élaborer un indice composite permettant d'évaluer le climat de discipline de leur établissement. 
Une corrélation nette avec la performance

Les déclarations des élèves sur le climat de discipline de leur établissement varient non seulement entre les pays, mais aussi entre les établissements eux-mêmes. En moyenne, dans les pays de I'OCDE, $15 \%$ de la variation des réponses des élèves à ces questions sont imputables à des différences entre les établissements ; en outre, en Estonie, en Italie, au Japon, en Lettonie, en République tchèque et en Slovénie, plus de $20 \%$ de la variation des réponses des élèves sont imputables à des variations inter-établissements.

Il existe une forte corrélation entre le climat de discipline en classe et la performance.

Le climat de discipline de la classe et de l'établissement peut également influer sur l'apprentissage. Les classes et les établissements qui connaissent davantage de problèmes de discipline sont moins propices à l'apprentissage, car les enseignants sont contraints de passer plus de temps à ramener I'ordre avant de pouvoir enseigner à proprement parler. Dans 61 pays et économies ayant participé à l'enquête PISA 2009, les élèves fréquentant un établissement où le climat en classe est plus propice à l'apprentissage tendent à obtenir de meilleurs résultats.

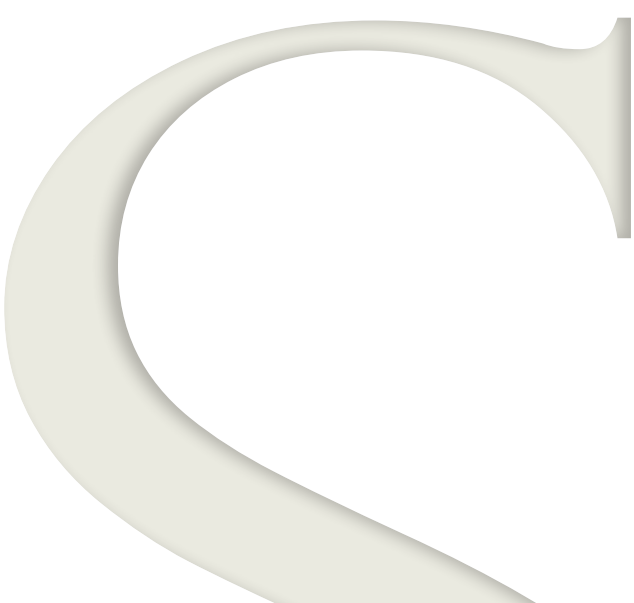

\begin{tabular}{|c|c|c|c|}
\hline \multirow[b]{2}{*}{$\begin{array}{l}\square \\
\square \begin{array}{l}\text { Corrélation } \\
\text { positive }\end{array} \\
\square \begin{array}{l}\text { Aucune } \\
\text { corrélation }\end{array} \\
\square \\
\begin{array}{l}\text { Corrélation } \\
\text { négative }\end{array} \\
\mathrm{m}: \text { données manquantes }\end{array}$} & \multicolumn{3}{|c|}{$\begin{array}{c}\text { Corrélation entre le climat de discipline moyen } \\
\text { de l'établissement et... }\end{array}$} \\
\hline & $\begin{array}{l}\text {... le statut } \\
\text { socio-économique } \\
\text { moyen des élèves } \\
\text { de l'établissement }\end{array}$ & $\begin{array}{c}\text {... la performance } \\
\text { des élèves en } \\
\text { compréhension } \\
\text { de l'écrit }\end{array}$ & $\begin{array}{c}\text {... la performance } \\
\text { des élèves en } \\
\text { compréhension de l'écrit } \\
\text { après contrôle du statut } \\
\text { socio-économique et du } \\
\text { profil démographique } \\
\text { des élèves et de } \\
\text { l'établissement, } \\
\text { et de diverses autres } \\
\text { caractéristiques de } \\
\text { l'établissement }\end{array}$ \\
\hline \multicolumn{4}{|l|}{ Australie } \\
\hline \multirow{2}{*}{\multicolumn{4}{|c|}{\begin{tabular}{|l|} 
Autriche \\
Belgique
\end{tabular}}} \\
\hline & & & \\
\hline \multicolumn{4}{|l|}{ Canada } \\
\hline \multirow{2}{*}{\multicolumn{4}{|c|}{\begin{tabular}{|l} 
Chili \\
République tchèque \\
\end{tabular}}} \\
\hline & & & \\
\hline \multicolumn{4}{|l|}{\begin{tabular}{|l|} 
République tchèque \\
Danemark \\
\end{tabular}} \\
\hline \multicolumn{4}{|l|}{\begin{tabular}{|l|} 
Estonie \\
Finlande \\
\end{tabular}} \\
\hline \multicolumn{4}{|l|}{ Finlande } \\
\hline France & & & $\mathrm{m}$ \\
\hline \multicolumn{4}{|l|}{ Allemagne } \\
\hline Grèce & & & \\
\hline Hongrie & & & \\
\hline Islande & & & \\
\hline Irlande & & & \\
\hline Israël & & & \\
\hline Italie & & & \\
\hline Japon & & & \\
\hline Corée & & & \\
\hline Luxembourg & & & \\
\hline Mexique & & & \\
\hline Pays-Bas & & & \\
\hline Nouvelle-Zélande & & & \\
\hline Norvège & & & \\
\hline Pologne & & & \\
\hline Portugal & & & \\
\hline République slovaque & & & \\
\hline Slovénie & & & \\
\hline Espagne & & & \\
\hline Suède & & & \\
\hline Suisse & & & \\
\hline Turquie & & & \\
\hline Royaume-Uni & & & \\
\hline États-Unis & & & \\
\hline Moyenne OCDE & & & \\
\hline Albanie & & & \\
\hline Argentine & & & \\
\hline Azerbaïdjan & & & \\
\hline Brésil & & & \\
\hline Bulgarie & & & \\
\hline Colombie & & & \\
\hline Croatie & & & \\
\hline Dubaï (EAU) & & & \\
\hline Hong-Kong (Chine) & & & \\
\hline Indonésie & & & \\
\hline Jordanie & & & \\
\hline Kazakhstan & & & \\
\hline Kirghizistan & & & \\
\hline Lettonie & & & \\
\hline Liechtenstein & & & $\mathrm{m}$ \\
\hline Lituanie & & & \\
\hline Macao (Chine) & & & $\mathrm{m}$ \\
\hline Monténégro & & & $\mathrm{m}$ \\
\hline Panama & & & \\
\hline Pérou & & & \\
\hline Qatar & & & \\
\hline Roumanie & & & \\
\hline Fédération de Russie & & & \\
\hline Serbie & & & \\
\hline Shanghai (Chine) & & & \\
\hline Singapour & & & \\
\hline Taipei chinois & & & \\
\hline Thaïlande & & & \\
\hline Trinité-et-Tobago & & & \\
\hline \begin{tabular}{|l|} 
Tunisie \\
\end{tabular} & & & \\
\hline Uruguay & & & \\
\hline
\end{tabular}

Remarque : les pays de l'OCDE sont indiqués en noir.

Source : Base de données PISA 2009 de l'OCDE ; Résultats du PISA 2009 : Les clés de la réussite des établissements d'enseignement : Ressources, politiques et pratiques, tableaux IV.4.2 et IV.2.14c.

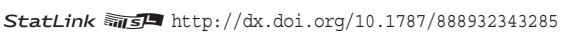


Les établissements qui bénéficient d'un meilleur climat de discipline présentent également d'autres caractéristiques étroitement liées à un meilleur niveau de performance. Ainsi, l'effectif d'élèves de ces établissements est en général issu d'un milieu plus favorisé. Dans 36 pays et économies, il existe une corrélation positive entre le climat de discipline de l'établissement et le statut socio-économique moyen de son effectif d'élèves.

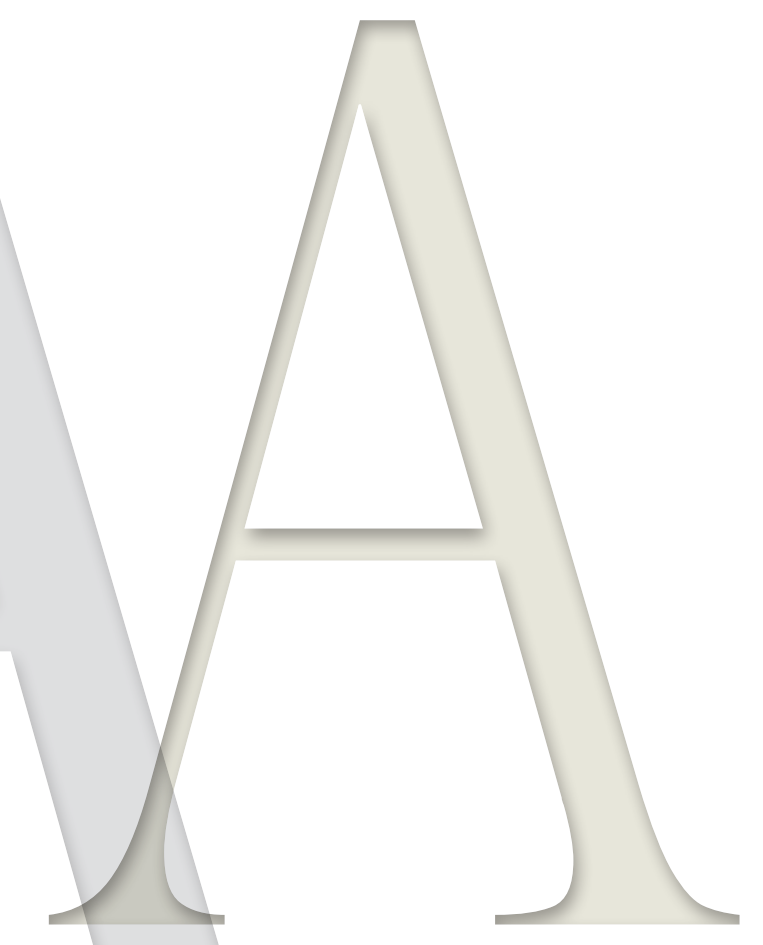

Toutefois, même après contrôle du statut socio-économique et du profil démographique des élèves et de l'établissement, ainsi que de diverses autres caractéristiques de l'établissement, telles que les ressources éducatives dont il dispose, son mode de gouvernance et les dispositifs d'évaluation et de responsabilisation qui y sont en place, dans 31 pays et économies, les établissements jouissant $d^{\prime}$ un meilleur climat de discipline tendent à obtenir de meilleures performances. Le climat de discipline est I'une des rares caractéristiques de niveau Établissement pour laquelle on observe, de façon systématique et dans tous les pays, une corrélation positive significative avec la performance, même après contrôle d'autres caractéristiques de l'établissement et du milieu des élèves. En outre, la forte corrélation observée entre le statut socio-économique des élèves et le climat de discipline laisse penser que l'impact du statut socio-économique sur la performance des élèves peut être atténué par un bon climat de discipline dans l'établissement.

Pour conclure: Un climat de discipline propice à l'apprentissage présente non seulement une corrélation positive avec la performance des élèves, mais les résultats du PISA suggèrent aussi qu'il peut atténuer l'impact du statut socio-économique des élèves sur la performance.

Pour tout complément d'information

Contacter Miyako Ikeda (Miyako.IKEDA@oecd.org)

Consulter OCDE (2011), Résultats du PISA 2009 : Les clés de la réussite des établissements d'enseignement : Ressources, politiques et pratiques, Volume IV, PISA, Éditions OCDE.

\begin{tabular}{ll}
\hline & Prochain numéro \\
$\begin{array}{l}\text { Voir } \\
\text { www.pisa.oecd.org }\end{array}$ & Elèves issus de l'immigration : \\
www.oecd.org/pisa/infocus & que peuvent-ils nous apprendre \\
& sur nos systèmes d'éducation? \\
\hline
\end{tabular}

